\title{
A Novel Non-DC Biased Intensity Modulated Indoor MIMO-VLC System Using Walsh Precoder
}

\author{
P. Muneer $\mathbb{D}^{1}{ }^{1}$ V. P. Thafasal Ijyas, ${ }^{1}$ Mohamed Abbas, ${ }^{1,2}$ Hassen Loukil, ${ }^{1,3}$ Ali Algahtani, ${ }^{4,5}$ \\ Amir Kessentini, ${ }^{4,6,7}$ and Abdul Wase Mohammed ${ }^{1}$ \\ ${ }^{1}$ Electrical Engineering Department, College of Engineering, King Khalid University, Abha 61413, Aseer, Saudi Arabia \\ ${ }^{2}$ Department of Computers and Communications, College of Engineering, Delta University for Science and Technology, Egypt \\ ${ }^{3}$ Electronics and Information Technology Laboratory, University of Sfax, National Engineering School of Sfax, Tunisia \\ ${ }^{4}$ Department of Mechanical Engineering, College of Engineering, King Khalid University, Abha, 61413 Asir, Saudi Arabia \\ ${ }^{5}$ Research Center for Advanced Materials Science (RCAMS), King Khalid University, P.O. Box 9004, Abha, 61413 Asir, Saudi Arabia \\ ${ }^{6}$ Laboratory of Electromechanical Systems (LASEM), National Engineering School of Sfax, University of Sfax, Route de Soukra km 4, \\ 3038 Sfax, Tunisia \\ ${ }^{7}$ Nabeul's Foundation Institute for Engineering Studies, University of Carthage, IPEIN, Nabeul 8000, Tunisia
}

Correspondence should be addressed to P. Muneer; mparayangat@kku.edu.sa

Received 24 October 2019; Revised 17 March 2020; Accepted 11 June 2020; Published 9 July 2020

Academic Editor: Pham Tien Dat

Copyright (c) 2020 P. Muneer et al. This is an open access article distributed under the Creative Commons Attribution License, which permits unrestricted use, distribution, and reproduction in any medium, provided the original work is properly cited.

\begin{abstract}
In this paper, we propose an indoor visible light communication (VLC) system which makes use of Walsh precoding and multiple light-emitting diodes (LEDs) at the transmitter for multiplexing the data. The proposed VLC system is based on intensity modulation and uses the notion of spatial modulation for communicating the positive and negative values of the synthesized transmit signal using signal processing technique at the transmitter. We also illustrate the flexibility, ability, and easiness of the system to configure well in a multiuser environment. We have also developed a near-maximum likelihood (ML) detection technique for efficiently decoding the data bits at the receiver. The proposed near-ML reduces the search space involved in ML technique by using the initial ZF solution. The results of the simulation studies illustrate that the proposed technique offers a higher data rate and good bit error rate (BER) performance in indoor VLC environments.
\end{abstract}

\section{Introduction}

Visible light communication (VLC) has recently emerged as a promising paradigm in wireless communication with multiple applications in various scenarios. There is a flurry of research bringing forth many innovative techniques. Notwithstanding this, there are many challenges that need to be addressed prior to the development and deployment of full-fledged systems. Of paramount importance is the development of techniques that can substantially increase the overall throughput of VLC systems. Spatial diversity through multiple-input, multipleoutput (MIMO) techniques can be used for this. An appropriate precoder and equalizer design is a concomitant to a MIMO VLC system design. This is an open problem, with many innovative solutions proposed recently in the related litera- ture. Recently, high-speed VLC systems with data rates of about $6.4 \mathrm{~Gb} / \mathrm{s}$ with MIMO have been achieved [1].

The system in [2] proposes a precoding technique for massive MIMO VLC systems. Successive interference cancellation (SIC) is used for the multiuser detection. Spatial multiplexing can be combined with WDM to achieve very high data rates [3]. Optical spatial modulation (OSM) or space shift keying (SSK) is such a technique [4]. A MIMO VLC system employing two-dimensional LED arrays at the transmitter and receiver is proposed in [5]. However, the field of view (FOV) and range of the systems need to be improved further.

Assignment of exponent for achieving channel diversity in nonorthogonal multiple-access (NOMA) for users with ill-conditioned channels is used in [6]. This method is not suitable for similar channels with similar condition numbers. 
To overcome this, another technique is proposed in [7]. This method makes use of precoding to generate different effective channel matrices for the various users. This method is suitable for massive MIMO deployments. However, the spatial correlation may become high as the number of transmitters and receivers is very large, if we disregard the optimal arrangement of transmitter LEDs and receiver photodiodes. The configuration in [8] is a promising solution to obtain only the dominant line of sight (LoS) component with significantly less correlation among the channels. In [8], the PDs are rotated $45^{\circ}$ in the $x y$-plane and tilted $60^{\circ}$ in the $y z$ -plane, which may lead to negligible multipaths and increase in LoS component [8]. A precoding matrix-based technique using constant amplitude zero auto correlation (CAZAC) sequences is used in [9]. A study and analysis of the effectiveness of precoding techniques for different VLC scenarios is presented in [10].

Energy-efficient precoding for multiuser, multiple-input single-output (MISO) VLC is the substance of [11]. The authors also propose an optimal precoding technique for a $4 \times 2$ multiuser system. A combination of Time Division Multiple Access (TDMA) and modified zero-forcing (ZF) is used to deal with the multiuser nonnegative interference in VLC systems in [12]. A joint precoder and equalizer design is dealt with in [13] also. A max-min approach to solve the signal-to-interference-noise ratio (SINR) is proposed in [14].

Many works are available in the literature which deal with multicarrier VLC systems. One popular technique is orthogonal frequency division multiplexing (OFDM) with a suitable modification for intensity modulation and direct detection (IM/DD) [15]. In OFDM-based VLC systems, the baseband signal modulates the intensity of the optical frequency carrier. Since the inverse discrete Fourier transform (IDFT) precoding at the transmitter in OFDM produces a complex signal which is not suitable for intensity modulation, we need to perform suitable modification at the transmitter side. One method is to use only half of the carriers and arrange the data symmetrically to obtain a real output at the output of the precoder. One of the drawbacks of using OFDM is that the output of IDFT is Gaussian distributed which may result in very low intensities frequently at the transmitter.

In most intensity-modulated multicarrier systems, DC biasing is used at the transmitter to convert the bipolar signal to a unipolar form. For example, in OFDM-based techniques, a DC bias is added to the resulting signal at the output of the IDFT precoder to convert the unipolar signal to a bipolar signal [16, 17]. A spectrally efficient precoding/decoding scheme for optical-OFDM (OOFDM) is detailed in [18]. It can be used for both single-input, single-output (SISO) and MIMO VLC systems. Two remarkable techniques which propose a VLC-compatible OFDM are non-DC biased optical (NDC) OFDM and indexed non-DC biased optical (INDC) OFDM techniques mentioned in [19]. A technique based on the singular value decomposition (SVD) for MIMO VLC system is mentioned in [20]. Also, a nonorthogonal multiple access- (NOMA-) based MIMO VLC system is presented in [21], which seeks to improve the achievable sum rate and power allocation in an indoor VLC systems. As mentioned before, the Gaussian-distributed output at the transmitter side in OFDM-based VLC systems results in very low intensities most of the time resulting in poor BER performance at low SNRs. Besides, special schemes are required in OFDM-based, intensity-modulated VLC systems for generating real signals at the transmitter [19].

In this paper, we propose an indoor visible light communication (VLC) system which makes use of the Walsh precoding and multiple light-emitting diodes (LEDs) at the transmitter for multiplexing the data. The highlights of the contribution are summarized as follows:

(i) We propose a VLC system that makes use of intensity modulation and use the notion of spatial modulation for communicating the positive and negative values of the synthesized transmit signal using signal processing techniques at the transmitter

(ii) The proposed system is highly flexible and easy to configure in both single and multiuser and environments

(iii) We have shown the performance of the proposed modulation and demodulation technique by using zero forcing (ZF) and maximum likelihood (ML) detection scheme at the receiver. Also, we developed a near-ML detection technique by reducing the search space involved in ML via initial ZF solution

(iv) The results of the simulation studies illustrate that the proposed technique offers a higher data rate and good bit error rate (BER) performance under indoor VLC environments

The rest of the paper is organized as follows: we give a description of the new signal design for the proposed intensity-modulated MIMO VLC system in Section 2. The details regarding the transmitter and receiver designs are given in Section 2.1 and Section 2.2, respectively. Section 3 details the equalization and data detection techniques. A near ML technique which makes use of the subset of search space is discussed in Section 4. Section 5 provides a detailed discussion on computational complexity. In Section 6 we have presented the simulation results with necessary related discussion. Section 7 gives the concluding remarks.

\section{Proposed Intensity Modulation Technique}

In this section, we present the proposed signal synthesis technique at the transmitter and the signal analysis technique used at the receiver for efficient communication of the data bits. Both the transmitter and receiver are equipped with multiple transmit and receive antennas, respectively. We have provided the details regarding the transmitter and the receiver in the subsections below.

2.1. Transmit Signal Synthesis Using Walsh Matrix and Spatial Intensity Modulation. We consider a MIMO-VLC system which makes use of intensity modulation for communication. The entire process of the synthesis of the transmit signal is expressed in the block diagram shown in Figure 1. We first 


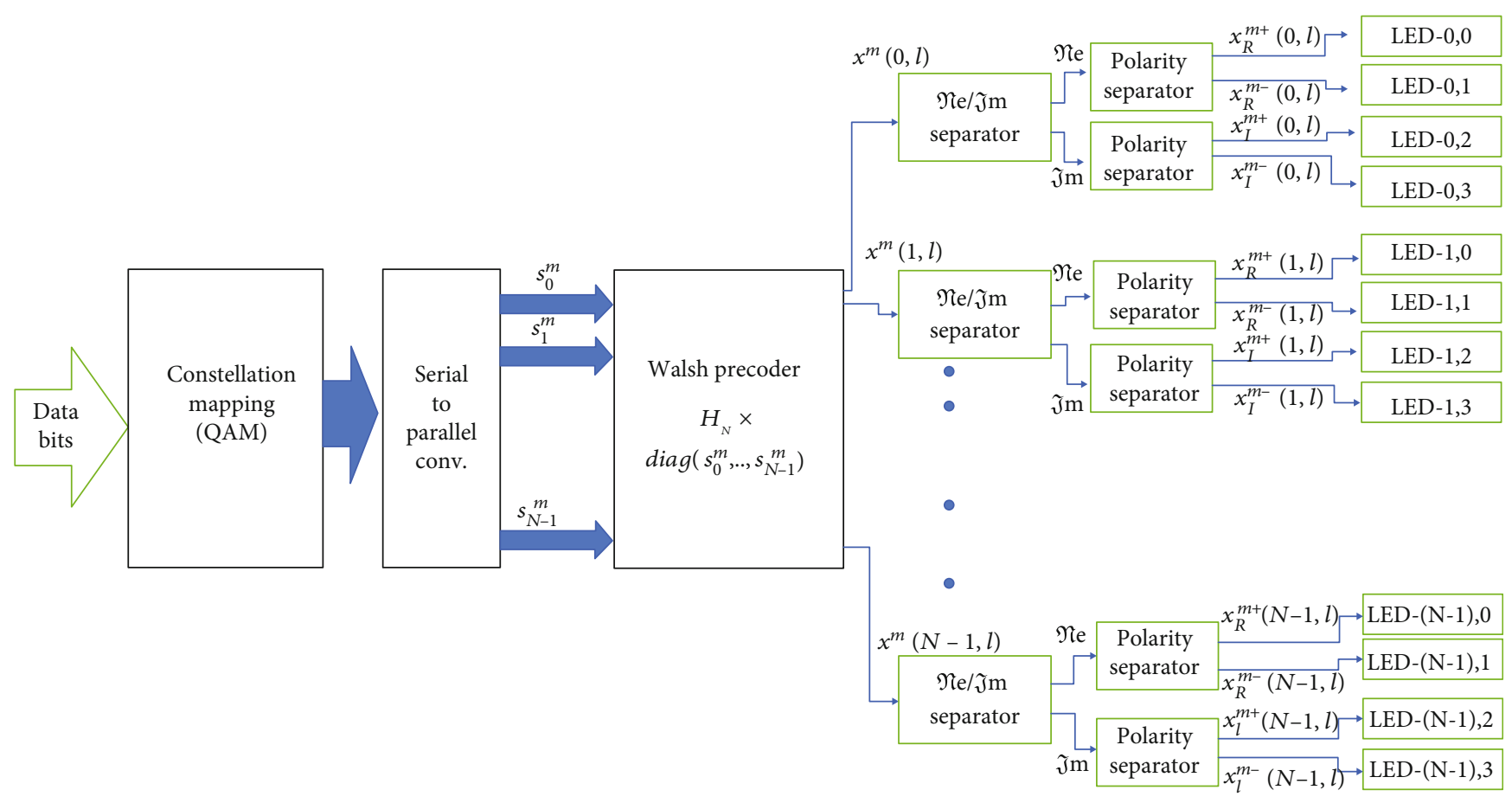

FIgURe 1: Transmit signal synthesis technique using Walsh precoder.

map $N \bar{M} M$ data bits into $N M$ quadrature amplitudemodulated (QAM) symbols. These symbols are then converted in to $N$ parallel streams, where each stream consists of $M$ symbols where $M$ is the number of symbols in each frame. Here, $\bar{M}$ is the number of data bits carried by each QAM symbol. The data frame vector in the $n^{\text {th }}$ parallel branch is given as

$$
\overline{\mathbf{s}}_{n}=\left[s_{n}^{0}, s_{n}^{1}, \cdots, s_{n}^{M-1}\right]^{T}
$$

where $\overline{\mathbf{s}}_{k}$ is the set of all symbols in the $n^{\text {th }}$ stream and $s_{k}^{m}$ denotes the $m^{\text {th }}$ symbol ofthe data frame in the $n^{\text {th }}$ parallel branch. We made use of the Walsh matrix of order $N$ to synthesize the transmit signal for the proposed VLC system [22]. We define the normalized matrix in a recursive form as

$$
\mathbf{H}_{k}=\frac{\sqrt{2(k-1)}}{\sqrt{k}} \mathbf{H}_{2} \otimes \mathbf{H}_{k-1},
$$

where we define

$$
\mathbf{H}_{2}=\frac{1}{\sqrt{2}}\left[\begin{array}{cc}
1 & 1 \\
1 & -1
\end{array}\right] .
$$

Here, $\otimes$ denotes the Kronecker product operation. By using the Walsh matrix, we obtain the first phase of the synthesized signal $\mathbf{X}$ by making use of all the $N$ parallel streams as

$$
\mathbf{X}=\mathbf{H}_{N} \mathbf{S}
$$

where we define $\mathbf{S}=\left[\operatorname{diag}\left(\mathbf{s}^{0}\right), \cdots, \operatorname{diag}\left(\mathbf{s}^{M-1}\right)\right]$ as a matrix of dimension $N \times N M$. Here, the vector $\mathbf{s}^{m}$ of length $N$ is defined as

$$
\mathbf{s}^{m}=\left[s_{0}^{m}, \cdots, s_{N-1}^{m}\right]^{T},
$$

where $s_{k}^{m} \in S$. With the above notion of $\mathbf{s}^{m}$, we can define the submatrices in matrix $\mathbf{X}$ as

$$
\mathbf{X}=\left[\mathbf{X}^{0}, \cdots, \mathbf{X}^{M-1}\right]
$$

where $\mathbf{X}^{m}=\mathbf{H}_{N} \times \operatorname{diag}\left(\mathbf{s}^{m}\right)$. The matrix $\mathbf{X}^{m}$ has the dimension $N \times N$. Let us define the $(n, l)^{\text {th }}$ entry of $\mathbf{X}^{m}$ as $x^{m}(n, l)$ $=x_{R}^{m}(n, l)+j x_{I}^{m}(n, l)$, where $j$ is defined as $\sqrt{-1}$. Note that each element in $\mathbf{X}^{m}$ has both real and imaginary parts. Here, we make use of the concept of spatial modulation to transmit the real and imaginary parts of the data which may contain both positive and negative polarities. As shown in Figure 1, a complex value is communicated by using a set of four LEDs. Let us consider the signal $x^{m}(n, l)$ in the $n^{\text {th }}$ parallel branch. The real part of this signal can be represented as

$$
x_{R}^{m}(n, l)=x_{R}^{m(+v e)}(n, l)+x_{R}^{m(-v e)},
$$

where we define $x_{R}^{m(+v e)}$ as the signal values with positive polarity and $x_{R}^{m(-v e)}$ as the signals with negative polarity. Similarly, the imaginary part can also be represented in the same way. To transmit the signal $x_{R}^{m}(n, l)$, we use the combination of two LEDs. For the $n^{\text {th }}$ branch, we have LEDs indexed as LED $-n, 0$ and LED $-n, 1$ for transmitting real part of the signal. If the signal $x_{R}^{m}(n, l)$ is positive, we transmit the signal 
$x_{R}^{m+}$ which is the same as $x_{R}^{m(+v e)}$ using the LED indexed as LED $-n, 0$. At the same time, LED $-n, 1$ transmits zero. However, we transmit $x_{R}^{m-}$ using the LED indexed by LED $-n, 1$, while the LED $-n, 0$ transmits zero at the same time. Here, we define $x_{R}^{m-}=-x_{R}^{m(-v e)}$. The above-mentioned procedure is true for the imaginary part also. The positive and negative amplitudes of the signal $x_{I}^{m}(n, l)$ are communicated through the $\mathrm{LED}-n, 2$ and $\mathrm{LED}-n, 3$, respectively, in the same way.

We use the vector $\mathbf{x}_{n, l}^{m}=\left[\mathbf{x}_{R}^{m}(n, l)^{T}, \mathbf{x}_{I}^{m}(n, l)^{T}\right]^{T}$ of length four, to represent the transmit signal vector at the $l^{\text {th }}$ instant of the $n^{\text {th }}$ branch of $m^{\text {th }}$ symbol in a frame, where we define.

$$
\mathbf{x}_{R}^{m}(n, l)=\left\{\begin{array}{cc}
{\left[\begin{array}{c}
x_{R}^{m+}(n, l) \\
0
\end{array}\right],} & \text { for } x_{R}^{m}(n, l) \geq 0 \\
{\left[\begin{array}{c}
0 \\
x_{R}^{m-}(n, l)
\end{array}\right],} & \text { for } x_{R}^{m}(n, l)<0
\end{array}\right\},
$$

which makes use of LED $-n, 0$ and LED $-n, 1$. Similarly, we have

$$
\mathbf{x}_{I}^{m}(n, l)=\left\{\begin{array}{cc}
{\left[\begin{array}{c}
x_{I}^{m+}(n, l) \\
0
\end{array}\right],} & \text { for } x_{I}^{m}(n, l) \geq 0 \\
{\left[\begin{array}{c}
0 \\
x_{I}^{m-}(n, l)
\end{array}\right],} & \text { for } x_{I}^{m}(n, l)<0
\end{array}\right\},
$$

which makes use of LED $-n, 2$ and LED $-n, 3$. Hence, the entire transmit vector at the $l^{\text {th }}$ instant of the $m^{\text {th }}$ symbol in a frame is given by

$$
\mathbf{x}_{l}^{m}=\left[\mathbf{x}_{0, l}^{m T}, \cdots, \boldsymbol{x}_{N-1, l}^{m}\right]^{T} .
$$

Note that

$$
\mathbf{x}_{n, l}^{m}=\left[\mathbf{x}_{R}^{m}(n, l)^{T}, \mathbf{x}_{I}^{m}(n, l)^{T}\right]^{T}
$$

$\mathbf{x}_{n, l}^{m}$ is a vector of dimension $\left(N_{t} \times 1\right)$, where $N_{t}=4 N$ is the total number of LEDs used at the transmitter. Since there are $N$ parallel channels with $\bar{M}$ bits in each channel, the total number of data bits transmitted through $N$ channels is given by $N \bar{M}$. With the above notion of transmission, the achieved data rate per frame of transmission of the proposed system is given by

$$
\mu=N \bar{M} M \text { bpf }
$$

Here, bpf is the number of bits per frame.

The proposed system is highly flexible to configure in a single user as well as a multiuser scenario and works well in both scenarios. It is a well-known fact that different sets of subcarriers of orthogonal frequency division multiplexing (OFDM) can also be allotted to different users in a multiple access scheme called OFDMA. The same kind of scheme is possible in the proposed technique also by allotting a set of indices from the pool of indices $\mathscr{I}=\{n=0,1, \cdots, N-1\}$ to each user. In a multiuser scenario, we can define the subindex set for each user as $\mathscr{I}_{i} \subset \mathscr{I}$, where $i=1,2, \cdots, K$ and $\bigcup_{i=1}^{K}$ $\mathscr{I}_{i}=\mathscr{I}$. Note that if a carrier index is not allotted to a particular user in a multiuser scenario, then the data modulated on it is zero. Using the above subindex allotment scheme, we can accommodate a maximum of $N$ users in the system. Hence, the use of Walsh precoding eases the process of multiplexing and multiple access in VLC systems.

2.2. Receive Signal Model. As mentioned in the previous section, the unipolar signal is transmitted over the MIMO-VLC channel. We define the channel matrix $\mathbf{G}$ with dimension $\left(N_{t} \times N_{r}\right)$, where $N_{r}$ is the number of photodetectors used at the receiver. The block diagram of the proposed receiver design is given in Figure 2. The details regarding equalization and data detection are provided in the following sections. The optical signal received at the PDs at the $l^{\text {th }}$ instant is given by

$$
\mathbf{y}_{l}^{m}=\mathbf{G} \mathbf{x}_{l}^{m}+\mathbf{n}_{l}^{m}
$$

where $\mathbf{y}_{l}^{m}$ received signal, $\mathbf{n}_{l}^{m}$ is the noise vector of dimension $\left(N_{r} \times 1\right)$, and $\mathbf{G}=r \overline{\mathbf{G}}$, with $r$ as the responsivity of the detector.

\section{Equalization and Data Detection}

In this section, we explain the equalization and the data detection technique for the proposed modulation technique. We first explain the ZF scheme and introduce the idea of ML. Further, a near ML solution is proposed to reduce the computational complexity.

3.1. Zero Forcing Equalization Scheme. The output of each PD is collected to form a vector $\mathbf{y}_{l}^{m}$ as shown in (13). We then apply the zero-forcing technique to obtain the equalized vector $\mathbf{z}_{l}^{m}$ as follows:

$$
\mathbf{z}_{l}^{m}=\mathbf{G}^{-1} \mathbf{y}_{l}^{m}
$$

where $\mathbf{z}_{l}^{m}=\left[\mathbf{z}_{0, l}^{m T}, \cdots, \mathbf{z}_{N-1, l}^{m}\right]^{T}$ is the ZF solution. Here, the vector $\quad \mathbf{z}_{n, l}^{m}=\left[\mathbf{z}_{R}^{m}(n, l)^{T}, \mathbf{z}_{I}^{m}(n, l)^{T}\right]^{T} \quad$ with $\quad \mathbf{z}_{R}^{m}(n, l)=$ $\left[z_{R, n, l}^{m}(0), z_{R, n, l}^{m}(1)\right]^{T}$ and $\mathbf{z}_{I}^{m}(n, l)=\left[z_{I, n, l}^{m}(0), z_{I, n, l}^{m}(1)\right]^{T}$. Since only one LED is activated at an instant, the SM detector is formulated based on the maximum received value. To perform the detection, we first calculate

$$
\begin{aligned}
\left|w_{R, n, l}^{m}\right| & =\max \left\{\left|z_{R, n, l}^{m}(0)\right|,\left|z_{R, n, l}^{m}(1)\right|\right\}, \\
\left|w_{I, n, l}^{m}\right| & =\max \left\{\left|z_{I, n, l}^{m}(0)\right|,\left|z_{I, n, l}^{m}(1)\right|\right\} .
\end{aligned}
$$

Once the magnitude of real and imaginary parts of received signals is estimated, it is necessary to assign the 


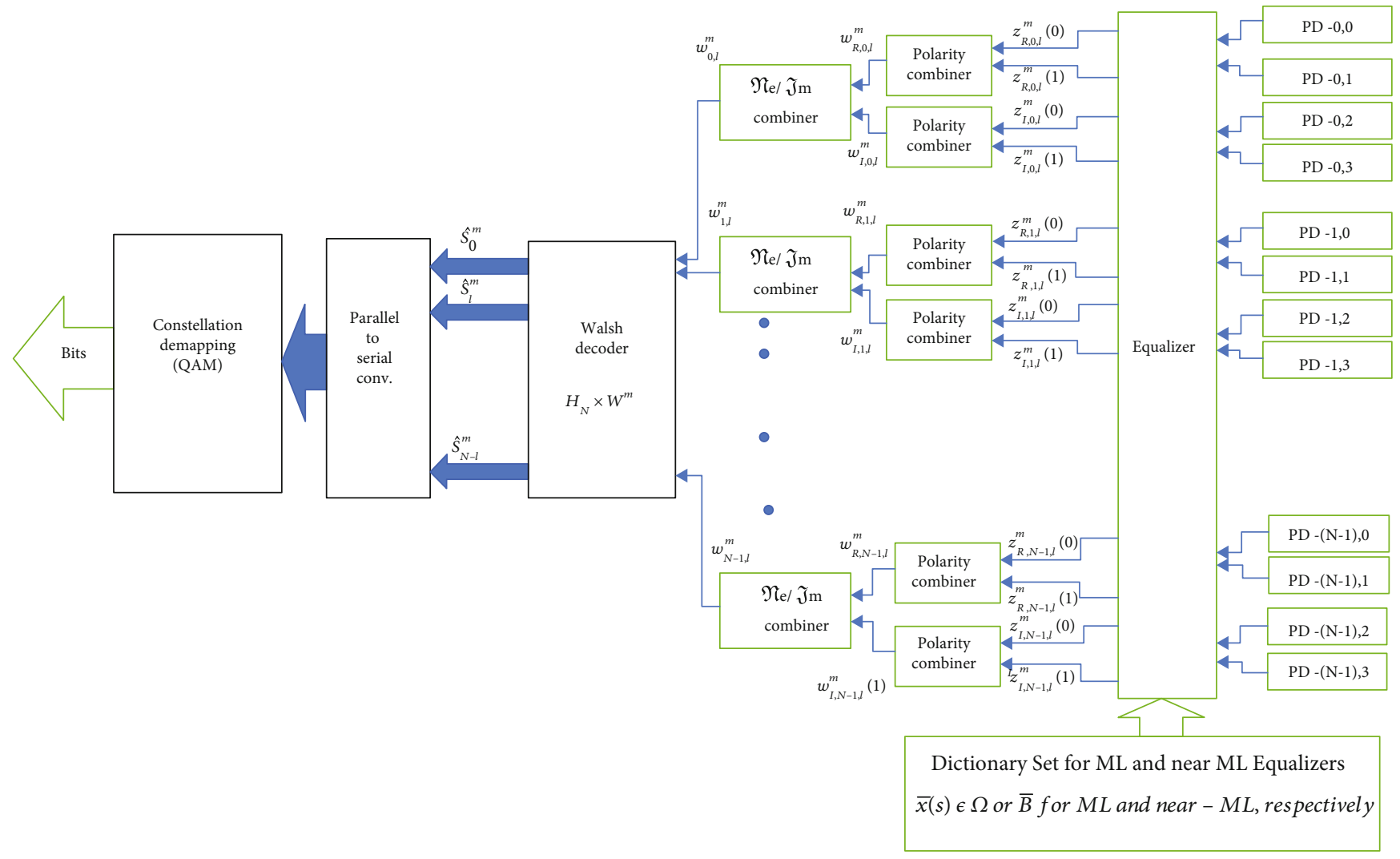

FIgURe 2: Proposed receiver design using multiple PDs.

polarities for them. The polarities of $w_{R, n, l}^{m}$ and $w_{I, n, l}^{m}$ are assigned as.

$$
\begin{aligned}
& \operatorname{sign}\left\{w_{R, n, l}^{m}\right\}=\left\{\begin{array}{lc}
+v e, & \text { if } \underset{p=0,1}{\operatorname{argmax}}\left|z_{R, n, l}^{m}(p)\right|=0 \\
-v e, & \text { if } \underset{p=0,1}{\operatorname{argmax}}\left|z_{R, n, l}^{m}(p)\right|=1
\end{array}\right\}, \\
& \operatorname{sign}\left\{w_{I, n, l}^{m}\right\}=\left\{\begin{array}{lc}
+v e, & \text { if } \underset{p=0,1}{\operatorname{argmax}}\left|z_{I, n, l}^{m}(p)\right|=0 \\
-v e, & \text { if } \underset{p=0,1}{\operatorname{argmax}}\left|z_{I, n, l}^{m}(p)\right|=1
\end{array}\right\},
\end{aligned}
$$

respectively. Here, sign $\{\bullet\}$ represents the polarity of received signal. Once the polarities are assigned, we obtain the equalized signal of the $n^{\text {th }}$ branch of the form

$$
w_{n, l}^{m}=w_{R, n, l}^{m}+j w_{I, n, l}^{m} .
$$

We collect all the equalized signals to form the vector

$$
\mathbf{w}_{l}^{m}=\left[w_{0, l}^{m}, \cdots, w_{N-1, l}^{m}\right]^{T} .
$$

Similarly, all the $N$ instances of the signal are processed and collected to obtain the matrix

$$
\mathbf{W}^{m}=\left[\mathbf{w}_{0}^{m}, \cdots, \mathbf{w}_{N-1}^{m}\right]^{T} .
$$

Once $\mathbf{W}^{m}$ is obtained, we can find the original equalized QAM symbol at the $n^{\text {th }}$ branch as

$$
\widehat{s}_{n}^{m}=\mathbf{H}_{N}(n,:) \mathbf{W}^{m}(:, n),
$$

where $\mathbf{H}_{N}(\mathrm{n},:)$ is the $n^{\text {th }}$ row of $\mathbf{H}_{N}$ and $\mathbf{W}^{m}(:, n)$ is the $n^{\text {th }}$ column of $\mathbf{W}^{m}$. Then, $\widehat{s}_{n}^{m}$ is demapped to obtain data bits.

3.2. Proposed ML Detection Technique. Since the channel gains in VLC systems are very low, there will be a significant enhancement of noise in the ZF scheme which in turn leads to poor BER performance at lower SNRs. For obtaining a better performance, we propose the use of ML detection techniques at the receiver. We first explain the proposed basic ML detection technique designed to decode the data at the receiver. Using the first principles from ML decoding technique, we have

$$
\mathbf{s} \wedge^{m}=\underset{\overline{\mathbf{x}}(s) ; s \in \Omega}{\arg \min }\left\{\left(\mathbf{y}_{l}^{m}-\mathbf{G} \overline{\mathbf{x}}(s)\right)^{T}\left(\mathbf{y}_{l}^{m}-\mathbf{G} \overline{\mathbf{x}}(s)\right)\right\},
$$

where $s \in \Omega$ and $\overline{\mathbf{x}}(s)$ is the vector of the form mentionedin (10). The set of all possible combinations of $\overline{\mathbf{x}}(s)$ can be precomputed and stored at the receiver and transmitter for the easiness. The simulation results show that the ML detection scheme offers a very good performance at lower SNRs also. However, the computational complexity is high when compared to the ZF scheme. The details regarding 
the computational complexities of the various schemes are discussed in the upcoming sections.

\section{Near ML-Based Detection Scheme for the Proposed Communication System}

In this section, we present a near ML-based detection scheme which extensively reduces the computational complexity of ML. To achieve this task, we make use of the ZF solution mentioned in (14). The ML formulation mentioned in (21) can also be rewritten as [23]

$$
\widehat{\mathbf{s}}^{m}=\underset{\overline{\mathbf{x}}(s) ; s \in \Omega}{\arg \min }\left\{\left(\overline{\mathbf{x}}(s)-\mathbf{z}_{l}^{m}\right)^{T} \mathbf{G}^{T} \mathbf{G}\left(\overline{\mathbf{x}}(s)-\mathbf{z}_{l}^{m}\right)\right\} .
$$

Note that $\mathbf{z}_{l}^{m}$ is the ZF solution computed using (14). After obtaining the ZF solution, the nearest neighbourhood of solutions is used to compute the reduced search space [24]. At the receiver, we precompute the set of all $\overline{\mathbf{x}}(s) \in \mathscr{B}$. Once the $\boldsymbol{z}_{l}^{m}$ is obtained, the reduced search space $\overline{\mathscr{B}}$ is computed by taking the $Q$ nearest neighbourhood of $\mathbf{z}_{l}^{m}$. By using the $Q$ nearest neighbourhoods, we obtain

$$
\widehat{\mathbf{s}}^{m}=\underset{\overline{\mathbf{x}}(s) \in \overline{\mathscr{B}}}{\arg \min }\left\{\left(\overline{\mathbf{x}}(s)-\mathbf{z}_{l}^{m}\right)^{T} \mathbf{G}^{T} \mathbf{G}\left(\overline{\mathbf{x}}(s)-\mathbf{z}_{l}^{m}\right)\right\} .
$$

Here, the cardinality of $\overline{\mathscr{B}}$ is $(Q+1)$.

\section{Computational Complexity}

In this section, we discuss the overall computational complexities of the proposed communication system. We can divide the entire system into two parts known as modulation and data detection. The total number of multiplications required at the transmitter and receiver for each frame is shown in Table 1 . Note that $\vartheta=Q+1$ is the cardinality of $\overline{\mathscr{B}}$.

\section{Simulation and Results}

In this section, we present the performance results for the proposed modulation technique for the VLC system. The parameters for the indoor VLC system considered in the simulation studies are given in Table 1. It is assumed that the PDs are kept symmetrical on the top of a table with respect to the centre of the floor with $d_{r x}$ of $0.1 \mathrm{~m}$. The LEDs are kept symmetrical with respect to centre of the room at $1 \mathrm{~m}$ apart and at $3 \mathrm{~m}$ height (i.e., $d_{t x}=1 \mathrm{~m}$ and $z=3 \mathrm{~m}$ ). Additional details regarding the channel modellingcan be found in [25]. The channel gain between $j^{\text {th }}$ LED and $i^{\text {th }} \mathrm{PD}$ is given as

$$
h_{i j}=\frac{A(\bar{n}+1)}{2 \pi R_{i j}^{2}}\left(\cos ^{\tilde{n}} \Phi_{i j} \cos \theta_{i j}\right)\left(\operatorname{rect}\left(\frac{\theta_{i j}}{F O V}\right)\right)
$$

where $A$ is the area of detector, $\theta_{i j}$ is the angle of incidence from $j$ - th LED to $i-$ th PD, $R_{i j}$ is the distance between $j-$ th LED and $i$-th PD, FOV is field view of the detector, $\operatorname{rect}(x)=1$ if $|x| \leq 1$ and $\operatorname{rect}(x)=0$ if $|x|>1$, and $\tilde{n}=-\ln$
TABLE 1: Computational complexity.

\begin{tabular}{lc}
\hline Method for equalization & Number of multiplications required \\
\hline ZF & $2 M N^{2}+M \mathcal{O}\left(N_{t}^{3}\right)$ \\
ML & $M\left(2+2^{\left(1+N \log _{2} \bar{M}\right)}\right) N^{2}+M \mathcal{O}\left(N_{t}^{3}\right)$ \\
Near ML & $M(2+9 M) N^{2}+M \mathcal{O}\left(N_{t}^{3}\right)$ \\
\hline
\end{tabular}

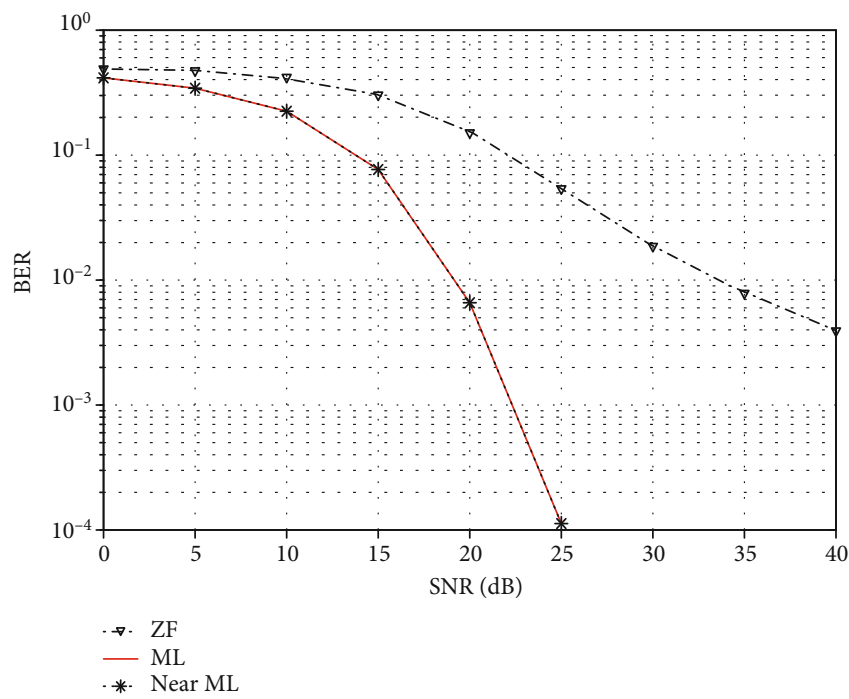

FIGURE 3: BER performance of the proposed system with $N=4$.

(2)/ln $\left(\Phi_{1 / 2}\right)$ is the mode number of the radiating lobe. Note that $\Phi_{1 / 2}$ is the half power semiangle of LED [25]. Also, the receivers are aligned to their correspondent LEDs to have stronger LoS link with a less correlation. It is a specially designed configuration to increase the line of sight component (LoS). In this design, the PDs are rotated $45^{\circ}$ in the $x y$ -plane and tilted $60^{\circ}$ in the $y z$-plane. Due to this effect, LoS component will be increased. A detailed analysis of this particular configuration is available in [3]. Also, the multiple LEDs can be configured at the different corners of the roof.

Figure 3 shows the BER performance of the proposed modulation scheme for a MIMO-VLC system with $N=4$ and $N_{r}=N_{t}=16$. It is found that the conventional $\mathrm{ZF}$ scheme offers good performance towards high SNRs and poor performance at lower SNR regimes. It is because of the noise amplification at lower SNRs in the ZF technique. However, it is evident from the results that the ML detection scheme offers a very good performance even at lower SNRs. Since the ML technique makes use of the complete search space, the computational complexity involved is very high. At the same time, the ZF technique needs high SNR for obtaining good BER performance. However, the proposed near-ML scheme uses ZF scheme to identify the search space. Since the proposed near-ML scheme performs the searching only in the modified search space defined using initial $\mathrm{ZF}$ solution, the complexity of near-ML is comparatively less to conventional ML scheme and guarantees good result. 


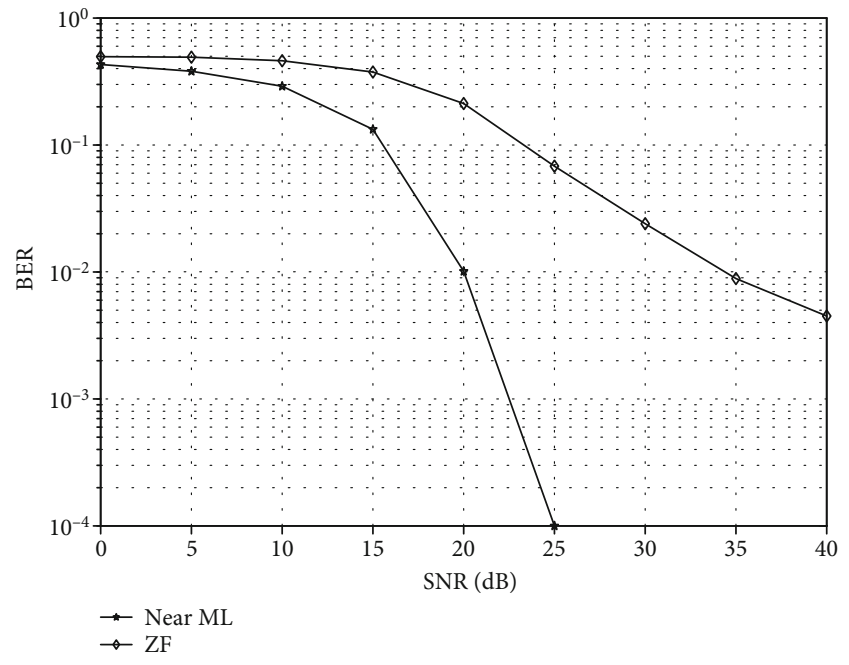

FIGURE 4: BER performance of the proposed system with $N=8$.

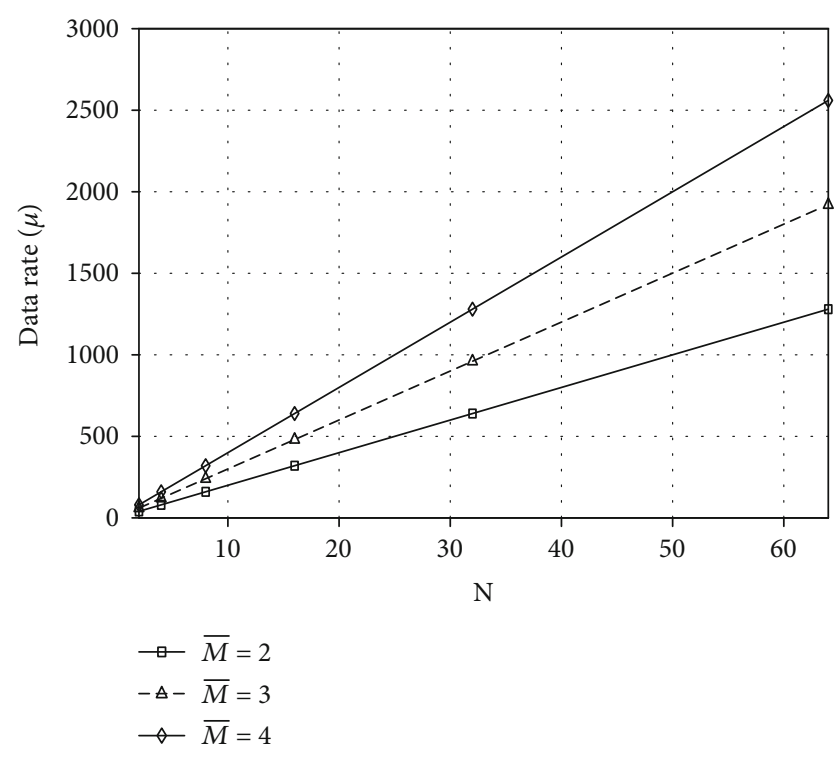

Figure 5: Variation of data rate $(\mu)$ in bpf with respect to $N$ for different constellations.

Figure 4 shows the BER performance of the proposed modulation scheme for a MIMO-VLC system with $N=8$ and $N_{r}=N_{t}=32$. The proposed system offers a very good BER performance. By doubling the value of $N$, the throughput is also doubled. By comparing with Figure 3, it is seen that the BER performance is not affected by increasing the throughput of the system.

Figure 5 shows the variation of data rate with respect to $N$. The throughput doubles when $N$ is doubled which shows a clear linear variation with respect to $N, M$, and $\bar{M}$.

Figure 6 illustrates the BER performance under a multiuser scenario with the number of users vary from two to sixteen with $N=16$. It is evident that the BER performance is not affected by this configuration. Note that the matrix $\mathbf{H}_{N}$ for the $u^{\text {th }}$ user is defined by the matrix $\mathbf{H}_{N}^{u}$, where the col-

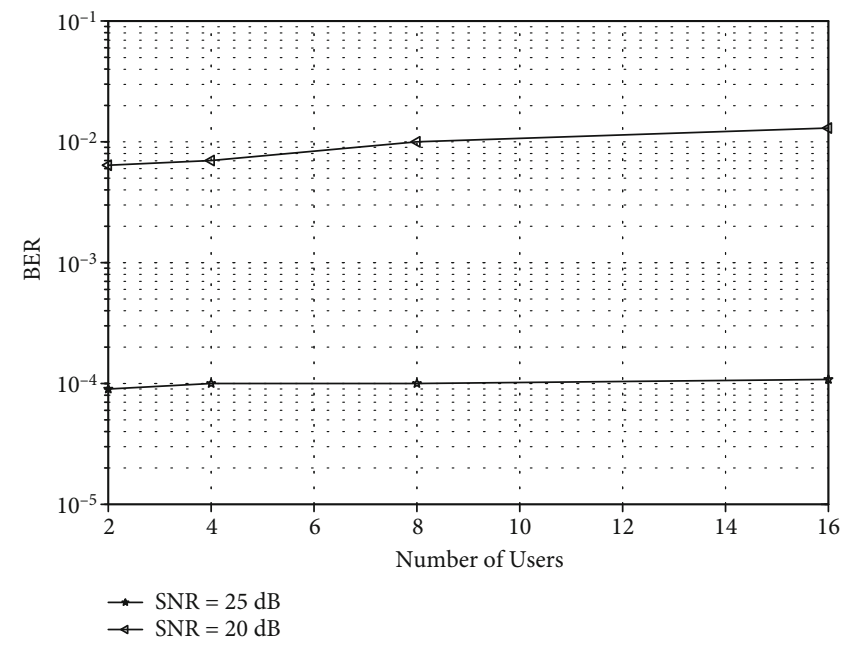

FIGURE 6: BER performance in a multiuser scenario with four active users with the number of users varying from two to sixteen.

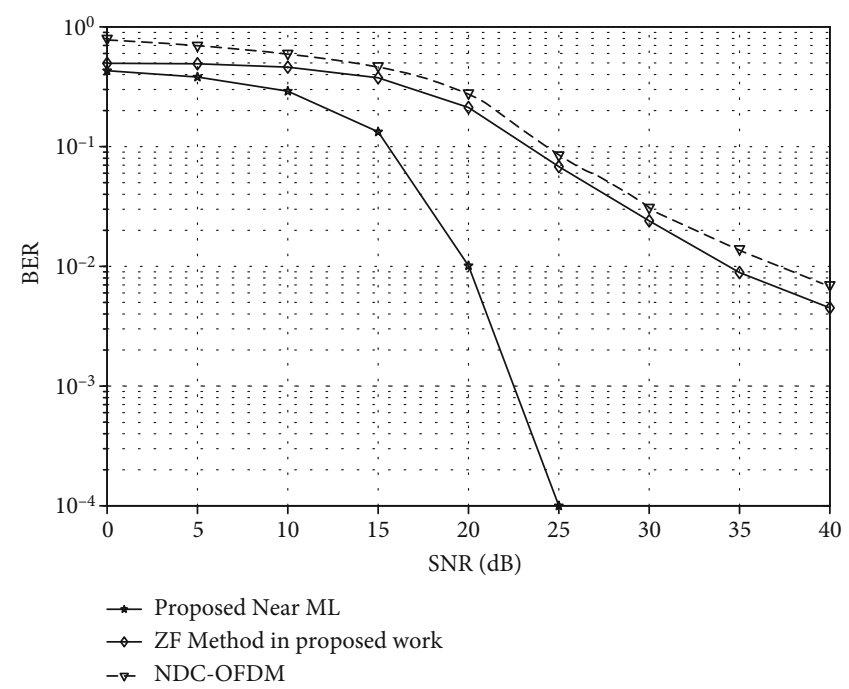

FIgURE 7: Performance comparison of the proposed work with NDC-OFDM in [19].

umns of $\mathbf{H}_{N}^{u}$ are zero vectors for the column indices corresponding to other active users. Hence, $\sum_{u=1}^{U} \mathbf{H}_{N}^{u}=\mathbf{H}_{N}$.

In Figure 7, we have compared the performance of the proposed scheme employing Walsh precoding with that of the NDC-OFDM-based intensity modulation given in [19]. It is found that the proposed techniques which use ZF detection outperform the NDC-OFDM technique. In higher SNR regimes, we obtain an SNR improvement of around $3 \mathrm{~dB}$ at a BER of $10^{-2}$. Also, it is to be noted that the proposed near-ML scheme gives a remarkable performance. This is because lowintensity signals at the output of transmitter which is the characteristic of NDC-OFDM are more or less completely eliminated due to Walsh precoding used in our scheme.

\section{Conclusion}

In this paper, we presented an indoor MIMO-VLC system which makes use of the Walsh precoding at the transmitter 
for multiplexing the data. The proposed VLC system uses intensity modulation and makes use of the notion of spatial modulation for communicating the positive and negative values of the synthesized transmit signal. The proposed system has the flexibility to configure well in a multiuser environment. We have presented the use of $\mathrm{ZF}$ and $\mathrm{ML}$ approaches in detecting/decoding the data also. A near-ML detection technique for efficiently decoding the data is also presented by reducing the search space in $\mathrm{ML}$ decoding by making use of an initial ZF solution. The results of the simulation studies illustrate that the proposed technique offers a good data rate and BER performance under indoor VLC environments.

\section{Data Availability}

The datasets supporting the results of this article are included in the article.

\section{Conflicts of Interest}

The authors declare that there is no conflict of interest regarding the publication of this paper.

\section{Authors' Contributions}

The authors contributed equally to this research. All authors read and approved the final manuscript.

\section{Acknowledgments}

The authors extend their special appreciation and thanks to the Deanship of Scientific Research at King Khalid University for funding this work through Research Groups Program under grant number R.G.P.2/35/40.

\section{References}

[1] I.-C. Lu, C.-H. Lai, C.-H. Yeh, and J. Chen, "6.36 Gbit/s RGB LED-based WDM MIMO visible light communication system employing OFDM modulation," in 2017 Optical Fiber Communications Conference and Exhibition (OFC), Los Angeles, CA, USA, 2017.

[2] R. Mitra and V. Bhatia, "Precoding technique for illconditioned massive MIMO-VLC system," in 2018 IEEE 87th Vehicular Technology Conference (VTC Spring), pp. 1-5, Porto, Portugal, June 2018.

[3] S. Rajbhandari, "Spatial and wavelength division multiplexing for high-speed VLC systems: an overview," in 2016 10th International Symposium on Communication Systems, Networks and Digital Signal Processing (CSNDSP), Prague, Czech Republic, July 2016.

[4] T. Fath and H. Haas, "Optical spatial modulation using colour LEDs," in 2013 IEEE International Conference on Communications (ICC), pp. 3938-3942, Budapest, Hungary, June 2013.

[5] S. Rajbhandari, J. J. D. McKendry, J. Herrnsdorf et al., "A multigigabit per second integrated multiple-input multiple-output VLC demonstrator," Journal of Lightwave Technology, vol. 35, no. 20, pp. 4358-4365, 2017.

[6] R. Mitra and V. Bhatia, "Precoded Chebyshev-NLMS-based pre-distorter for nonlinear LED compensation in NOMA-
VLC," IEEE Transactions on Communications, vol. 65, no. 11, pp. 4845-4856, 2017.

[7] S. Jain, R. Mitra, and V. Bhatia, "Adaptive precoding-based detection algorithm for massive MIMO visible light communication," IEEE Communications Letters, vol. 22, no. 9, pp. 1842 1845, 2018.

[8] A. Yesilkaya, E. Basar, F. Miramirkhani, E. Panayirci, M. Uysal, and H. Haas, "Optical MIMO-OFDM with generalized LED index modulation," IEEE Transactions on Communications, vol. 65 , no. 8, p. 1, 2017.

[9] J. Ma, J. He, Q. Chen et al., "A MB-CAZAC precoding combined with 128/64/32/16-QAM modulation for OFDM-VLC system," Optics Communications, vol. 424, pp. 154-158, 2018.

[10] T. Jiang, D. Liu, M. Tang et al., "Investigation of DC-biased optical OFDM with precoding matrix for visible light communications: theory, simulations, and experiments," IEEE Photonics Journal, vol. 10, no. 5, pp. 1-16, 2018.

[11] Z. Y. Wang, H. Y. Yu, and D. M. Wang, "Energy-efficient precoding design for multiuser MISO VLC systems based on joint detection," IEEE Access, vol. 7, pp. 16274-16280, 2019.

[12] B. Li, J. Wang, R. Zhang, H. Shen, C. Zhao, and L. Hanzo, "Multiuser MISO transceiver design for indoor downlink visible light communication under per-LED optical power constraints," IEEE Photonics Journal, vol. 7, no. 4, pp. 1-15, 2015.

[13] K. Ying, H. Qian, R. J. Baxley, and S. Yao, "Joint optimization of precoder and equalizer in MIMO VLC systems," IEEE Journal on Selected Areas in Communications, vol. 33, no. 9, pp. 1949-1958, 2015.

[14] H. Sifaou, K. H. Park, A. Kammoun, and M. S. Alouini, "Optimal Linear Precoding for Indoor Visible Light Communication System," in 2017 IEEE International Conference on Communications (ICC), Paris, France, May 2017.

[15] M. Z. Afgani, H. Haas, H. Elgala, and D. Knipp, "Visible light communication using OFDM," in 2nd International Conference on Testbeds and Research Infrastructures for the Development of Networks and Communities, 2006. TRIDENTCOM 2006, pp. 129-134, Barcelona, Spain, March 2006.

[16] M. F. Sanya, L. Djogbe, A. Vianou, and C. Aupetit-Berthelemot, "DC-biased optical OFDM for IM/DD passive optical network systems," Journal of Optical Communications and Networking, vol. 7, no. 4, pp. 205-214, 2015.

[17] A. A. Sathar, P. Muneer, V. P. T. Ijyas, M. Usman, M. Z. M. Shamim, and M. Shiblee, "Filter-bank modulation based signal design and transmission techniques for intensity modulated MIMO visible light communication systems," Wireless Personal Communications, vol. 111, no. 2, pp. 1129-1150, 2020.

[18] S. M. Hardan, O. Bayat, and A. A. Abdulkafi, "A new precoding scheme for spectral efficient optical OFDM systems," Optics Communications, vol. 419, pp. 125-133, 2018.

[19] S. P. Alaka, T. L. Narasimhan, and A. Chockalingam, "Coded index modulation for Non-DC-biased OFDM in multiple LED visible light communication," in 2016 IEEE 83rd Vehicular Technology Conference (VTC Spring), Nanjing, China, July 2016.

[20] P. M. Butala, H. Elgala, and T. D. C. Little, "SVD-VLC: A novel capacity maximizing VLC MIMO system architecture under illumination constraints," in 2013 IEEE Globecom Workshops (GC Wkshps), pp. 1087-1092, Atlanta, GA, USA, December 2013.

[21] C. Chen, W.-D. Zhong, H. Yang, and P. Du, "On the performance of MIMO-NOMA-based visible light communication 
systems," IEEE Photonics Technology Letters, vol. 30, no. 4, pp. 307-310, 2018.

[22] C. Yubao and S. Zhongtao, "Application of walsh matrix in encoding laser beam for pointing multi-target," in Eighth Int. Conf. Electron. Meas. Instruments ICEMI'2007, Xi'an, China, October 2007.

[23] U. Fincke and M. Pohst, "Improved methods for calculating vectors of short length in a lattice, including a complexity analysis," Mathematics of Computation, vol. 44, no. 170, p. 463, 1985.

[24] H. Artés, D. Seethaler, and F. Hlawatsch, "Efficient detection algorithms for MIMO channels: a geometrical approach to approximate ML detection," IEEE Transactions on Signal Processing, vol. 51, no. 11, pp. 2808-2820, 2003.

[25] J. R. Barry, J. M. Kahn, W. J. Krause, E. A. Lee, and D. G. Messerschmitt, "Simulation of multipath impulse response for indoor wireless optical channels," IEEE Journal on Selected Areas in Communications, vol. 11, no. 3, pp. 367-379, 1993. 